\section{Pustulosis subcórnea (enfermedad de Sneddon-Wilkinson) Caso clínico}

\author{
YESENIA VALENZUELA ${ }^{1}$, CONSTANZA RAMÍREZ2,a, ENRIQUE BELLOLIO ${ }^{3}$
}

\section{Subcorneal pustular dermatosis. Report of one case}

We report a 35-year-old female patient with a one year history of a pustular and painful erythematous dermatitis, located in great folds, pubis and abdomen. She was evaluated in primary health care, receiving antifungal treatment, antimicrobials, topical and systemic non-steroidal anti-inflammatory drugs, with no response. A skin biopsy was compatible with subcorneal pustular dermatosis. She was initially treated with prednisone $(0.8 \mathrm{mg} / \mathrm{kg})$, observing a partial response. Therefore clotrimoxazole was initiated, obtaining an excellent response after 30 days of treatment.

(Rev Med Chile 2012; 140: 633-636).

Key words: Dermatitis; Skin diseases, eczematous; Trimethoprin-Sulfamethoxazole combination.

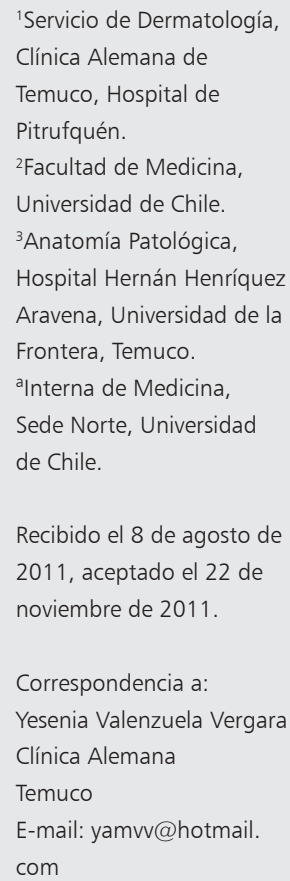

L as dermatosis neutrofílicas son un grupo de desórdenes, caracterizados por lesiones cutáneas en que el examen histológico revela intenso infiltrado inflamatorio en epidermis y dermis, compuesto principalmente por neutrófilos sin evidencia de infección ${ }^{1}$.

Dentro de éstas, se encuentra la dermatosis pustular subcórnea (Enfermedad de SneddonWilkinson) la que fue descrita por primera vez en $1956^{2}$. Se trata de una erupción crónica, relativamente benigna y recurrente caracterizada por pústulas flácidas agrupadas en un patrón anular sobre piel normal o eritematosa en pliegues axilares y/o inguinales, tronco y extremidades proximales ${ }^{3}$.

Es un cuadro clínico poco frecuente, con reportes esporádicos a nivel internacional, sin casos nacionales reportados. A continuación se presenta un caso clínico y una revisión de la literatura.

\section{Caso clínico}

Paciente femenina, 35 años. Antecedentes de hipotiroidismo en tratamiento con levotiroxina.
Presenta cuadro de un año de evolución caracterizado por pápulo-pústulas muy dolorosas, algunas confluentes, formando placas eritematosas con márgenes descamativos en grandes pliegues, pubis y abdomen (Figura 1A y 1B). Consulta en atención primaria de salud (APS) recibiendo múltiples tratamientos, antimicóticos tópicos y sistémicos, cefadroxilo y ketoprofeno sin respuesta.

Evaluada por médico internista, es derivada urgente a dermatólogo, dado el dolor invalidante que manifestaba la paciente.

Se sospecha pénfigo Hailey-Hailey indicándose hospitalización y biopsia. El estudio histopatológico de las lesiones identificó pústulas subcorneales con abundante infiltrado inflamatorio polimorfonuclear neutrófilo y epidermis con espongiosis leve compatible con una pustulosis subcórnea (Figura 2). Se inició tratamiento con corticoides endovenosos y analgesia; con posterior cambio a corticoides orales (Prednisona $0,8 \mathrm{mg} / \mathrm{Kg} / \mathrm{peso}$ ). Al mes de tratamiento presentó disminución parcial del eritema, descamación y dolor; sin embargo, continuaban apareciendo nuevas lesiones, por lo que se mantuvo la dosis durante otro mes. $\mathrm{Al}$ 
nuevo control, persistían pápulas eritematosas y escasas pústulas, asociadas a leve ardor y prurito (Figura 1C y 1D). Se decidió iniciar cotrimoxazol forte $(160 / 800 \mathrm{mg})$ un comprimido cada $12 \mathrm{hrs}$ por 10 días y luego un comprimido al día por 20 días. Al completar tratamiento, la paciente presentó excelente respuesta, sólo hiperpigmentación residual y refería estar completamente asintomática (Figura 1E y 1F). Se solicitó glucosa-6-fosfato deshidrogenasa para iniciar dapsona, sin embargo, la respuesta terapéutica a esta sulfa, tras dos meses de uso, fue inferior a la observada con el uso del cotrimoxazol, por lo que actualmente continúa con este último antibiótico.

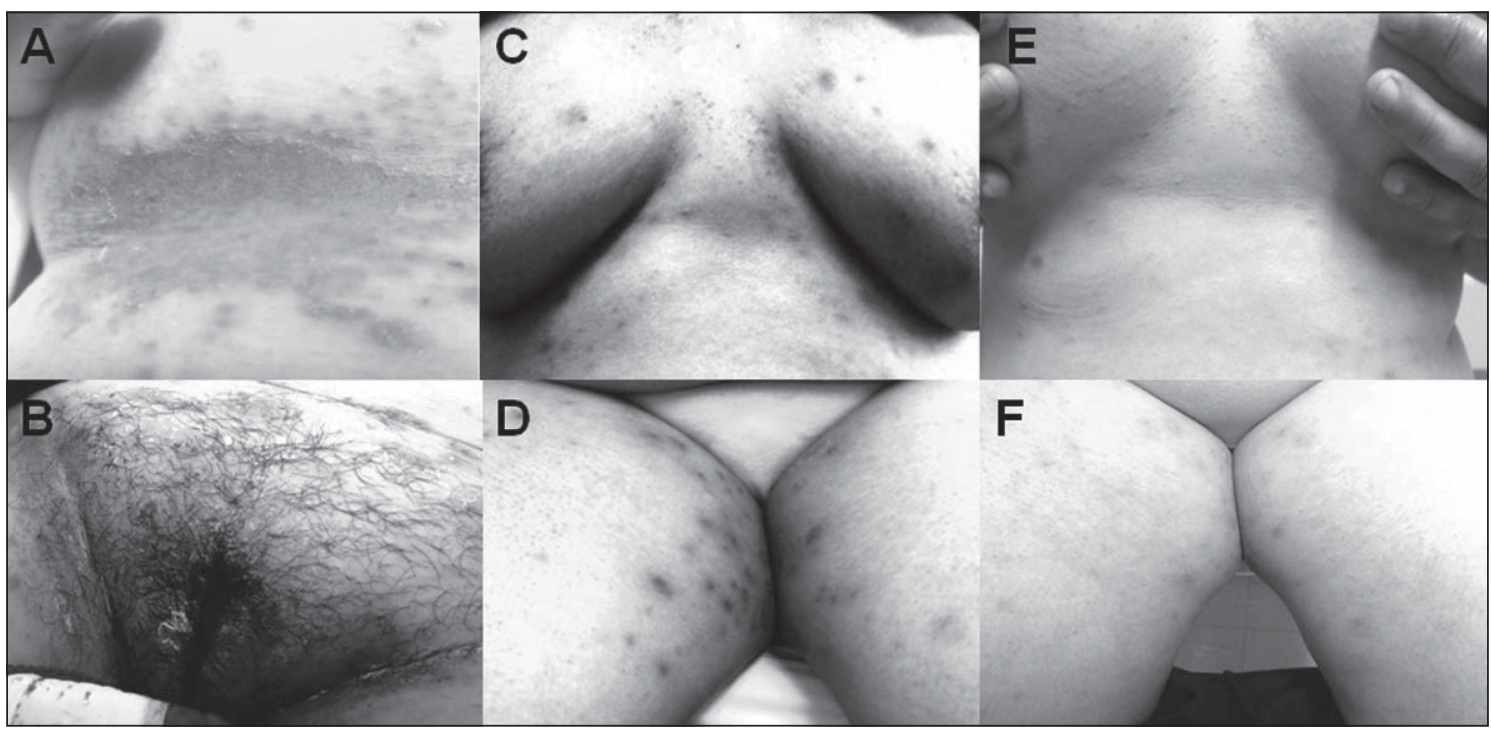

Figura 1. Fotografía clínica. A y B: Dermatosis eritematosa y pustular en pliegue submamario, inguinal y pubis. C y D: Después de 2 meses de uso de terapia corticoidal. E y F: Tras 30 días de tratamiento con cotrimoxazol forte donde se observa resolución de las lesiones.

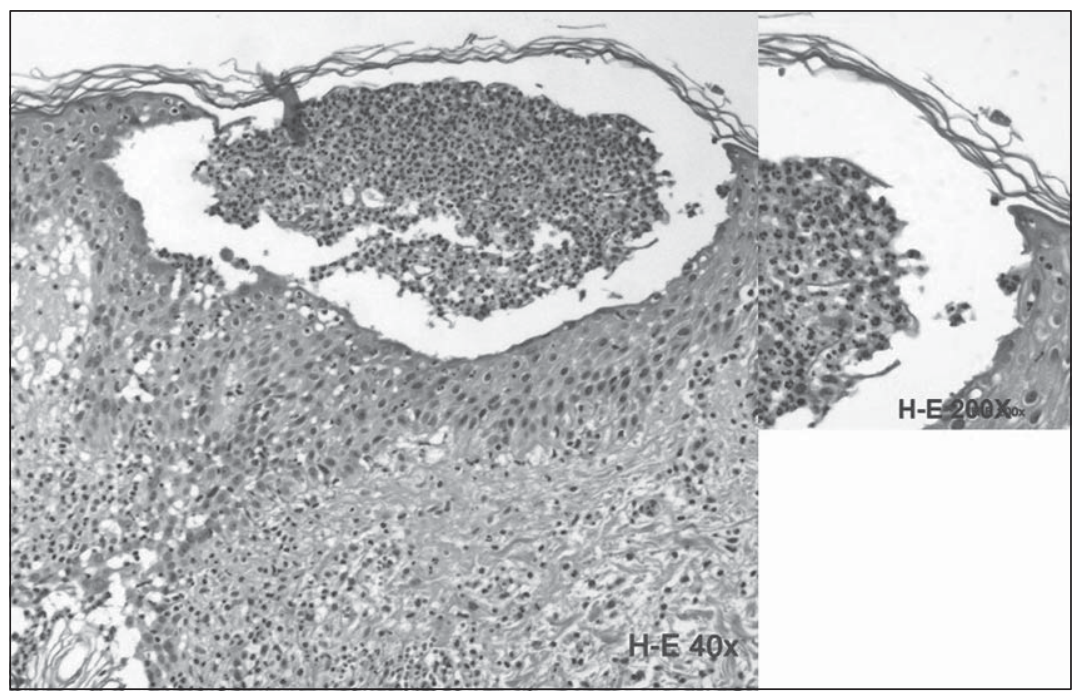

Figura 2. Fotografía histológica de lesiones donde se observa estrato corneo con ortoqueratosis, destacando en zona central la presencia de pústula subcorneal con abundante infiltrado inflamatorio polimorfonuclear neutrófilo, epidermis con espongiosis leve y exocitosis focal de neutrófilos, dermis papilar con escaso infiltrado inflamatorio linfocitario de distribución perivascular y presencia polimorfonucleares neutrófilos. 
Pustulosis subcórnea. Enfermedad de Sneddon-Wilkinson - Y. Valenzuela et al

\section{Discusión}

La dermatosis pustular subcórnea es un desorden poco frecuente, sin datos en la literatura sobre su real incidencia o prevalencia, que afecta con mayor frecuencia a mujeres entre los 40 y 60 años ${ }^{3,4}$.

La patogenia de este desorden aún es desconocida, sin embargo, se ha descrito una hiperactivación de los neutrófilos cutáneos que sería causada por una excesiva producción de factor de necrosis tumoral-alfa ${ }^{5}$.

Clínicamente los pacientes presentan una erupción con pústulas flácidas de varios milímetros de diámetro, sobre una piel normal o eritematosa. Las áreas más comúnmente afectadas son los pliegues como axilas, ingle, cuello, región submamaria y región proximal de extremidades. Se acompaña de prurito, dolor y no es habitual la presencia de signos sistémicos, ni compromiso de palmas, plantas, cara y mucosas ${ }^{3,4}$.

Las pústulas pueden encontrarse aisladas o agrupadas y tienden a coalecer en un patrón anular, circinado o serpiginoso. Estas son superficiales y se rompen fácilmente, dejando una costra superficial ${ }^{3}$.

Una vez que las lesiones se resuelven, habitualmente persiste una hiperpigmentación postinflamatoria. La enfermedad es relativamente benigna, pero usualmente tiene un curso crónico recurrente ${ }^{3,4}$.

Se han descrito en la literatura, múltiples enfermedades asociadas a este desorden como; gammapatía monoclonal por $\operatorname{IgA}^{6}$, desórdenes mieloproliferativos como mieloma múltiple ${ }^{7}$ y pioderma gangrenoso ${ }^{8}$. Reportes esporádicos los han asociado a artritis reumatoide ${ }^{9}$, enfermedad inflamatoria intestinal ${ }^{10}$, síndrome de Sjögren ${ }^{11}$, hipotiroidismo, hipertiroidismo, lupus eritematoso sistémico, esclerosis múltiple, crioglobulinemia, entre otros.

Dentro de los estudios diagnósticos se incluyen: el cultivo bacteriano y micológico los que son útiles para el diagnóstico diferencial y resultarán negativos. La electroforesis de proteínas resulta de utilidad en algunos casos, debido a la asociación reportada con gammapatías monoclonales IgA, como también IgG, las que podrían preceder en años a la aparición de un mieloma múltiple ${ }^{7}$.

La histopatología clásicamente revela una acumulación subcórnea de neutrófilos; sin embargo,
Tabla 1. Diagnóstico diferencial

\begin{tabular}{|l|}
\hline Impétigo \\
\hline Psoriasis pustular \\
\hline Intertrigo \\
\hline Pénfigo foliaceus \\
\hline Pénfigo por IgA \\
\hline Pustulosis exantemática aguda generalizada \\
\hline Eritema necrolítico migratorio \\
\hline Pustulosis amicrobiana en los pliegues \\
\hline Dermatitis herpetiforme \\
\hline Dermatofitosis \\
\hline Foliculitis eosinofilica pustular \\
\hline
\end{tabular}

este hallazgo no es específico y puede encontrarse en otros desórdenes como la psoriasis pustular, pustulosis exantemática aguda generalizada, pénfigo foliaceus, impétigo y dermatofitosis. En la pustulosis subcórnea, a diferencia de la psoriasis pustular, la epidermis presenta mínima espongiosis. Además, la dermis muestra infiltrado perivascular de neutrófilos, ocasionalmente monocitos, eosinófilos y la acantolisis no es predominante. La inmunofluorescencia directa e indirecta, habitualmente, es negativa ${ }^{13}$. $1^{4,13}$

El diagnóstico diferencial es múltiple. Tabla

El tratamiento de primera línea es la dapsona (sulfona) la que ha sido utilizada exitosamente; demostrándose efectos inmunomoduladores mediante inhibición de quimiotaxis, mieloperoxidasa, enzimas lisosomales y especias reactivas de oxígeno. Además, suprimiría la adherencia de los neutrófilos a IgG o IgA en la membrana basal epidérmica y a la albúmina ${ }^{14}$. Terapias alternativas incluyen sulfapiridina, retinoides (acitretina y etretinato), PUVA, fototerapia UVB de banda estrecha, corticoides, colchicina e inhibidores de factor de necrosis tumoral-alfa. Sin embargo, al igual que el caso presentado, se han descrito casos resistentes a estas terapias los que representan un desafío terapéutico ${ }^{15}$.

El cotrimoxazol (sulfametoxazol-trimetropim) es un antibiótico que combina una sulfonamida con un bacteriostático derivado de la trimetoxibenzilpirimidina. En conjunto inhiben la síntesis de ácido fólico y también se le han descrito pro- 
piedades inmunomoduladoras relacionadas con el bloqueo de la conversión de folatos en folinatos, inhibición de la autoinmunidad mediada por células, disminución de radicales libres y efectos antiinflamatorios. Ha sido utilizado en otros desórdenes inmunes como granulomatosis de Wegener, enfermedad de Crohn, colitis ulcerosa y artritis reumatoide ${ }^{16}$. Sin embargo, aún no se describen resultados en el tratamiento de estas dermatosis pustulares. El caso presentado mostró una intensa y rápida mejoría con el uso del cotrimoxazol, por lo que sería una valiosa alternativa terapéutica, con ventajas en cuanto a costo y accesibilidad. No obstante, se requiere la validación de estos resultados mediante la recopilación de un mayor número de casos y la realización de ensayos clínicos.

\section{Referencias}

1. Callen J. Neutrophilic dermatoses. Dermatol Clin 2002; 20: 409.

2. Sneddon I, Wilkinson D. Subcorneal pustular dermatosis. Br J Dermatol 1956; 68 (12): 385-94.

3. Cheng S, Edmonds E, Ben-Gashir M, Yu R. Subcorneal pustular dermatosis: 50 years on. Clin Exp Dermatol 2008; 33: 229-33.

4. Reed J, Wilkinson J. Subcorneal pustular dermatosis. Clin Dermatol 2000; 18: 301-13.

5. Grob J, Mege J, Capo C, Jancovicci E, Fournerie J, Bongrand $\mathrm{P}$, et al. Role of tumor necrosis factor-alfa in Sneddon-Wilkinson subcorneal pustular dermatosis. A model of neutrophil priming in vivo. J Am Acad Dermatol 1991; 25: 944-7.

6. Kasha E, Epinette W. Subcorneal pustular dermatosis
(Sneddon-Wilkinson disease) in association with a monoclonal IgA gammopathy: a report and review of the literature. J Am Acad Dermatol 1988; 19: 854.

7. Villasante A, Hormaechea J, García M, Vera E, Gilsanz C: Pustulosis of Sneddon-Wilkinson disease and multiple myeloma. An Med Intern 2001; 18 (7):373-5.

8. Kohl P, Hartschuh W, Tilgen W, Frosch P. Pyoderma gangrenosum followed by subcorneal pustular dermatosis in a patient with IgA paraproteinemia. J Am Acad Dermatol 1991; 24: 325.

9. Butt A, Burge S. Sneddon-Wilkinson disease in association with rheumatoid arthritis. Br J Dermatol 1995; 132: 313-5.

10. Delaporte E, Colombel J, Nguyen-Mailfer C, Plette F, Cortot A, Bergoend H. Subcorneal pustular dermatosis in a patient with Crohn's disease. Acta Derm Venereol (Stockh) 1992; 72: 301-2.

11. Tsuruta D, Matsumura A, Ishii M. Subcorneal pustular dermatosis and Sjogren's syndrome. Int J Dermatol 2005; 44: 55-7.

12. Todd D, Bingham E, Walsh M, Burrows D. Subcorneal pustular dermatosis and IgA paraproteinaemia: response to both etretinate and PUVA. Br J Dermatol 1991; 125: 387.

13. Razera F, Silveira G, Rangel R. Neutrophilic dermatosesPart II. An Bras Dermatol 2011; 86 (2): 195-211.

14. Modschiedler K. Dapsone and colchicine inhibit adhesion of neutrophilic granulocytes Arch Dermatol Res 2000; 292: 32-6.

15. Laifaoui J, Guillen E, Worret W, Ring J A case of subcorneal pustular dermatosis (Sneddon-Wilkinson-disease) not responding to dapsone: therapeutic alternatives. Acta Dermatoven APA 2003; 12 (3): 109-11.

16. Rozin A Cotrimoxazole treatment for rheumatoid arthritis. Semin Arthritis Rheum 2001; 31 (2): 133-41. 